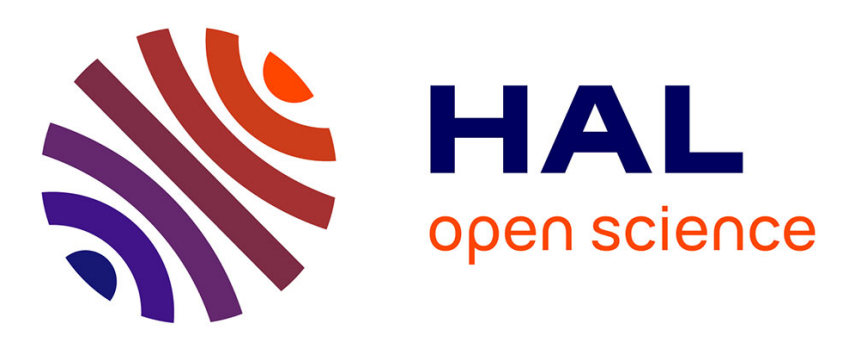

\title{
Vibrotactile guidance for trajectory following in computer aided surgery.
}

\author{
Jeremy Bluteau, Marie-Dominique Dubois, Sabine Coquillart, Edouard \\ Gentaz, Yohan Payan
}

\section{- To cite this version:}

Jeremy Bluteau, Marie-Dominique Dubois, Sabine Coquillart, Edouard Gentaz, Yohan Payan. Vibrotactile guidance for trajectory following in computer aided surgery.. Conference proceedings: .. Annual International Conference of the IEEE Engineering in Medicine and Biology Society. IEEE Engineering in Medicine and Biology Society. Annual Conference, 2010, 1, pp.2085-8. 10.1109/IEMBS.2010.5626270 . hal-00540744

\section{HAL Id: hal-00540744 \\ https://hal.science/hal-00540744}

Submitted on 29 Nov 2010

HAL is a multi-disciplinary open access archive for the deposit and dissemination of scientific research documents, whether they are published or not. The documents may come from teaching and research institutions in France or abroad, or from public or private research centers.
L'archive ouverte pluridisciplinaire HAL, est destinée au dépôt et à la diffusion de documents scientifiques de niveau recherche, publiés ou non, émanant des établissements d'enseignement et de recherche français ou étrangers, des laboratoires publics ou privés. 


\title{
Vibrotactile guidance for trajectory following in Computer Aided Surgery
}

\author{
Jeremy Bluteau*†॰ ${ }^{\dagger}$, Marie-Dominique Dubois ${ }^{\otimes}$, Sabine Coquillart ${ }^{\dagger}$, Edouard Gentaz ${ }^{\circ}$ and Yohan Payan*
}

\begin{abstract}
Most conventional computer-aided navigation systems assist the surgeon visually by tracking the position of an ancillary and by superposing this position into the $3 D$ preoperative imaging exam. This paper aims at adding to such navigation systems a device that will guide the surgeon towards the target, following a complex preplanned ancillary trajectory. We propose to use tactile stimuli for such guidance, with the design of a vibrating belt. An experiment using a virtual surgery simulator in the case of skull base surgery is conducted with 9 naïve subjects, assessing the vibrotactile guidance effectiveness for complex trajectories. Comparisons between a visual guidance and a visual+tactile guidance are encouraging, supporting the relevance of such tactile guidance paradigm.
\end{abstract}

\section{INTRODUCTION}

Computer-Aided Surgery (CAS) has become an accepted way of assisting surgeons during complex surgical interventions. These navigation systems provide an on-line tracking of surgical tools (or "ancillaries", often tracked by stereo cameras and markers) which positions are superimposed into previously acquired medical investigation such as computer tomography (CT), magnetic resonance imaging (MRI) or ultrasound images (US). The use of imaging technologies leads to possible $3 \mathrm{D}$ reconstructions and preoperative planning, depending on the medical intervention.

Most conventional CAS systems inform the surgeon about the distance of the ancillary relative to anatomical structures as well as preplanned data (safety areas, tumor, predefined trajectory,...). This information is usually provided through visualization devices (a screen) located beside the operative field, two or three meters away fron the surgeon. Quite recent researches tried to improve this visual feedbacks by (1) simplifying the amount of guidance information or (2) using another perception channel. In the first class of improvements, some punction applications like kidney biopsies or computer assisted systems for pedicle screw insertion display visual guidance by aligning crosses [7], [5]. These applications are restricted to straight trajectories. The second class of improvements propose to use another sensory channel for such guidance. Auditory channels have been extensively used, mainly because of their temporal efficiency [8]. However, their omnipresence as physiological variables

This work was supported by Région Rhônes-alpes, Cluster ISLE, PPF "interaction multimodale", France.

* Corresponding authors :

Jeremy.Bluteau@inrialpes.fr - Yohan.Payan@imag.fr

* TIMC Laboratory - Faculté de Médecine, Pavillon Taillefer, 38706 La Tronche cedex, France

$\dagger$ INRIA Grenoble Rhône-Alpes - LIG, Montbonnot, 38330 Saint Ismier, France

- LPNC laboratory - Grenoble University, 38400 Saint martin d'hères, France

$\otimes$ ENT department, CHU Saint Etienne, Hôpital Nord, 42000 Saint Etienne, France alarms dissuades their use for on-line continuous guidance. Recently, researchers have tried to use a still non-overloaded sensory modality namely the tactile channel. Due to the large amount of sensitive receptors on the tongue, researchers have shown that guidance of surgical gestures such as biopsies or target hit can be achieved by electro-tactile stimulation of the tongue thanks to a Tongue Display Unit [11], [10], [9]. Even if efficiency have been demonstrated, efforts on ergonomics, discomforts and pattern of information coding are still needed [10]. Other researches focused on the stimulation of the skin by vibrations. Vibrators can actually be placed on the dorsal face of the hand [2], or mounted on a belt, worn on the abdomen, torso or forearm [8]. Brell et al. [2] proposed a four vibrators tactile glove tracked by stereo cameras which indicates the correct position of the surgical tool. Results showed a less than $5 \mathrm{~mm}$ error to the target but problems of overshooting and oscillations during the trajectory following task. $\mathrm{Ng}$ et al. [8] demonstrated that tactile modality leads to higher reaction time compare to auditive stimulation but far lower reaction time compare to visual modality. Our project is more generally dedicated to a better characterization of the influence of these vibrotactile modalities onto the human performance during complex, online, and non straight trajectories following tasks. Since it is a surgical gesture that needs quite complex trajectories path to access the target, skull base surgery was choosed for our study.

\section{MATERIAL AND METHODS}

\section{A. Vibrotactile feedbacks}

The proposed solution is composed by vibrators, mounted on a belt worn by the participants around the abdomen. The control of vibration motors is provided by an USB controller card and relays (www.phidgets.com). Two design configurations were proposed, in order to experiment two different guidance paradigms. The first design (Belt1) is composed by 6 tiny vibrators $(\varnothing 6 \mathrm{~mm})$ which can run with two vibration levels ( $\approx 5000 \mathrm{rpm}$ and $\approx 2000 \mathrm{rpm}$ ). The activation of one of the 6 relays stands for the motor selection, the two last relays stand for the selection of the level of vibration (realized by adding resistors). The second design (Belt2) is composed by 8 vibrators whith a single level of vibration $(\approx 5000 \mathrm{rpm})$. In that case, two mounting belts are used, each composed by four motors. The vibration motors of belts 1 and 2 are manually arranged around the abdomen of the subjects to fit their anatomy, depending on the guidance paradigm used (see below). For development purposes, not encapsulated motors are used and firmly mounted on the belt with the secure 
Velcro attachment. Of course, for a real use in a surgical environments, encapsulated sterilizable motors will have to be designed.

\section{B. Guidance paradigms}

Two guidance paradigms associated to the two belts use (Belt1 or 2) were proposed. Our objective was to evaluate whether participants were more efficiently guided by one belt or the other one, suggesting different mental spatial representations of the task.
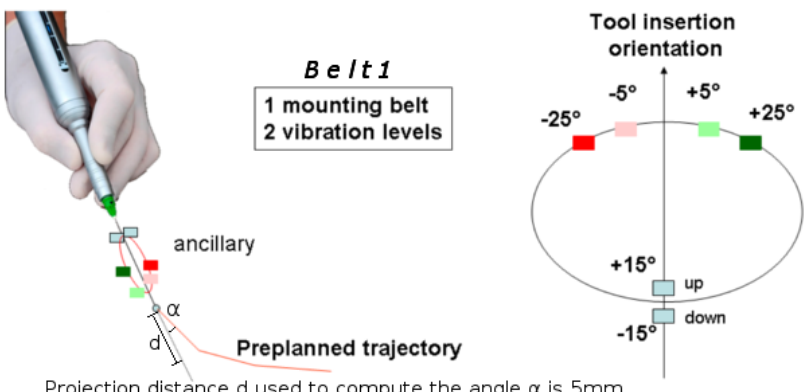

Projection distance $d$ used to compute the angle $\alpha$ is $5 \mathrm{~mm}$
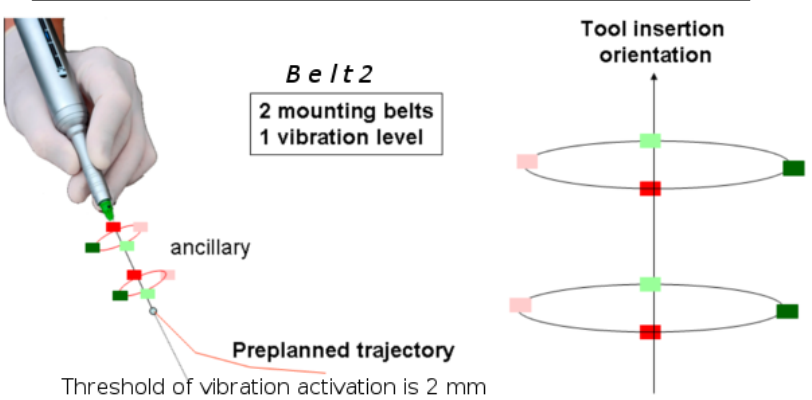

Fig. 1. The two proposed guidance paradigms. Top (configuration Belt1): the spatial representation of the tool is such as the tool is going from the back to the abdomen. Bottom (configuration Belt2): the spatial representation of the tool is surrounded by the two belts.

1) Guidance paradigm 1: This guidance paradigm is composed by 4 frontal vibrators located on the anterior side of the Belt1, indicating side and horizontal changes (yaw) in the orientation of the tool. Each vibrator is activated sequentially, depending on the angles figured in fig. 1 (top). The level of vibration is given by the difference between the current tool orientation and the planned trajectory. If the difference is higher than half of the triggering threshold then the vibration level is high, else it is set to low. Two vibrators are placed at the back of the belt, standing for the pitch orientation of the tool. They are also sequentially activated but in parallel with the frontal vibrators. When the tool is perfectly aligned with the planned trajectory, no vibration is given to the user.

2) Guidance paradigm 2: This second guidance paradigm is based on the 8 vibrators of Belt 2 ( $c f$. figure 1, bottom). Two sets of four motors are located on the cardinal points of the two mounting belts. The higher belt stands for the proximal position of the tool (close to the hand of the surgeon), while the lower belt stands for the extremity of the tool (distal position). The activation of one of the four vibrators of each mounting belt is sequential but activation from the lower and the higher mounting belts is possible simultaneously. Activations are based on difference of the tool position and orientation to the theoretical trajectory, starting with a distance of $0.02 \mathrm{~mm}$. The same yaw and pitch information are then possible.

3) Coding schemes: In addition, two schemes for direction coding are proposed, for both belts: demonstration coding, where the belt indicates the orientation to provide to the tool to be aligned with the theoritical planned trajectory; or correction coding, where the belt indicates the current alignment errors. Theses coding schemes are related in the literature as "direction coding" and "avoiding coding" [9].

\section{Surgery simulator}

To reproduce a situation similar to the actual skull base surgery and in order to quantitatively evaluate the tactile guidance, we decided to develop a virtual surgery simulator. Indeed, experiments on real anatomical pieces introduce a large amount of logistical and ethical problems (access to CT and MRI for the preoperative images, cadaver experiments). On the other hand, the uniqueness of those configurations that can not be validated by several experts or do not allow statistical analysis of large scale was not suitable for our purpose. Achieving a specific head phantom, dedicated to this experience revealed to be technically too complex and anatomically too simplistic regarding the structures to reproduce. Therefore, we used real anatomical data from a patient to build a simulator of the surgical gesture. This solution allowed us to repeat the experiment many times, with different trajectories, despite the lack of realism inherent in this type of simulators [4]. A general overview of the simulator is pictured in fig. 2. After registration of the preoperative data (CT and MRI), a 3D model of the patient's head based on iso surfaces generation was computed. This data set was used for the display of anatomical sections as in real computer-navigated surgery and was also used for the simulation rendering. This second role can be decomposed in two main parts: haptic rendering and visualization. Haptic force feedbacks were required for the realism of the simulation, otherwise the participants would not have been able to feel any contact with the virtual model, and therefore would not have been able to reproduce the surgery. Forces and contacts between virtual structures and surgical tools are restored to the user by a PHANToM Omni device (www.sensable.com). Stiffness values were linearly derived between 0 and $2 \mathrm{~N}$ from the voxels intensity of the preoperative CT exam. It is important to note here that two voxels with similar intensities rendered the same stiffness haptically. Even if these values were approximated, they allow real differences in the touch of differents structures such as bones, soft tissues, cavities, and vessels. During a real skull base surgery, the surgeon has to drill into the patient head bones. Simulating such interaction is still a complex issue [4] and has been solved by implementing the method proposed by [3], [6]. We strongly encourage the reader to refer to [1, chap. 8] for implementation details. 


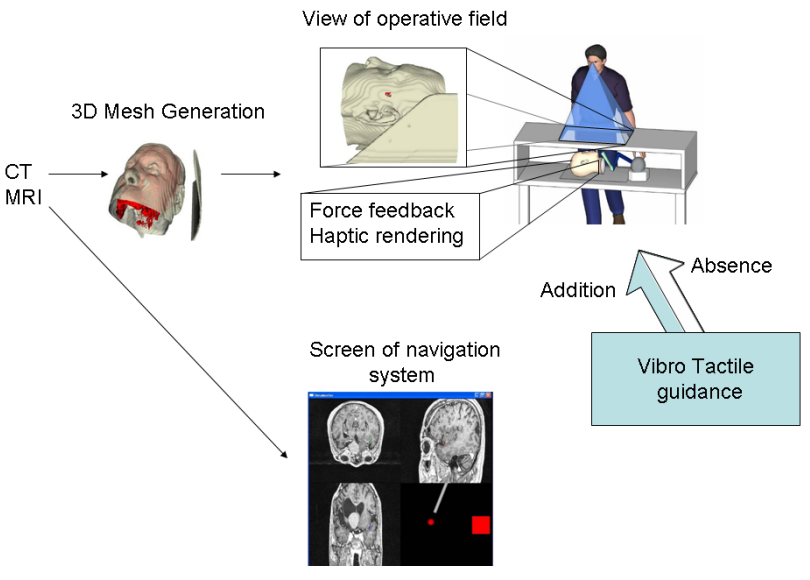

Fig. 2. Virtual skull base surgery simulator. From the preoperative data (CT and MRI), anatomical sections are displayed on a navigation system, and a 3D model of the patient is built. This model is haptically rendered by an haptic device (PHANToM Omni) placed below a computer screen where the simulation is visually rendered. The user sees on the screen the result of his co-located manipulation. The position of the haptic tool tip is sent to the navigation system to display the current anatomical slices to the user.

\section{Trajectory planning}

The trajectory used for this experiment was based on the planning defined by the surgeon, regarding the location of the tumor in relation to the surrounding anatomical structures of the patient's head. A specific application was developed to enable the surgeon to visualize a merged volume from CT and MRI data, and then to determine the theoretical trajectory from the entry point to the tumor. This work was done with an ENT surgeon from the Saint Etienne Hospital, France. The final trajectory used to evaluate our tactile guidance was composed by a series of 6 straight lines, passing dorsally to the left eye orbit, and was about $10 \mathrm{~cm}$ long.

\section{E. Experiment evaluation}

To evaluate the effects of tactile guidance, we designed an experiment based on the previously described surgery simulator, with different conditions.

1) Experimental conditions: The introduction of two tactile guidance paradigms (Belt1 and Belt2) and the fact that two coding schemes (Demonstration and Correction) exist led us to define four guidance conditions (Belt1-D, Belt1C, Belt2-D, Belt2-C) and one control condition where no tactile guidance was given (Visual only). Within each of these conditions, participants had visual feedbacks on the screen of the navigation system, with the $2 \mathrm{D}$ display of the the sagittal, axial and coronal CT slices corresponding to their tool tip current position.

2) Experimental protocol: Since we proposed two tactile guidance paradigms, specific trainings were needed. Two experiments were therefore defined. The first one acted as a familiarization step and allowed us to determine for each subject his/her preferences regarding guidance paradigms and coding schemes. This first experiment was based on a simplified training software where the user had to follow the previously defined trajectory $(c . f . \S \mathrm{D})$ with visual indications of the localization of vibrations. After a familiarization phase with each conditions, the participant gave us his/her preference for a coding scheme for both belt types. Then the performance of the user was assessed in a within-subjects design involving three randomized 6 trials blocks.

In the second experiment, we tested for the surgery task the effect of the best tactile guidance condition compared to the control condition. The same protocol was applied: a training phase was followed by a within-subjects performance assessment of two randomized 6 trials blocks.

3) Experimental measurements: To evaluate the performances of the participants during both experiments, the current position of the tool tip provided by the haptic device was recorded at $100 \mathrm{~Hz}$. Four criterions were choosen: duration and mean velocity of the complete trial (from the beginning to the end of theoretical trajectory), mean error and maximum error (computed from distances between experimental and theoretical trajectories).

During the second experiment, the trials were videotaped in order to determine the amount of time the participant spent looking at the navigation screen. This gave us another criterion which stands for the use of visual information during the task: the percentage of time spent looking at the navigation system screen.

4) Participants: Because of the busy schedule of surgeons and the duration of the complete experiment (about $2 \mathrm{~h}$ ), we decided to test first beginners population (i.e. naïve subjects). Ten subjects accepted to participate (age: $30.3 \pm 4$; only males). A subject was nevertheless excluded from the study because of a recording error. The present study was conducted in accordance with the Declaration of Helsinki and was approved by the local ethic committee. It was carried out with the understanding and the written consent of each participant which was obtained before the experiment.

\section{RESULTS}

\section{A. Experiment 1}

After the training phase, subjects had to choose a coding scheme (either demonstration or correction) for each belt type. None of the participants choose the Belt1 with a correction coding or the Belt 2 with a demonstration coding. We had therefore only three conditions to test during the performance assessment: Belt1-D, Belt2-C and Visual Only. For each measured criterion (raw data are summed up in the table I), an ANalysis Of VAriance (ANOVA) in repeated measurements ( 3 conditions x 6 trials) was computed. The influence of the order was not significant (as expected by the experimental protocol design) except for the mean error. Further analysis of this last criterion was cancelled. For maximum error criterion, a significant effect $(F(2,16)=$ $11.0 ; p<.01)$ was found between conditions. NewmansKeuls post-hocs tests detailed this effect by showing a significant difference between conditions Belt1-D and Belt2$\mathrm{C}(p<.01)$; and a significant difference between conditions Belt1-D and Visual Only $(p<.01)$. No significant difference was found between Belt2-C and Visual Only $(p>.25)$. Concerning duration, the ANOVA revealed no significant effect between conditions $(p>.25)$. Finally, 
dealing with mean velocity criterion, a significant effect was found $(F(2,16)=5.54 ; p<.05)$ between conditions. Post hocs tests (Newmans-Keuls) revealed significant differences between Belt1-D and Visual Only.

TABLE I

SUMMARY OF RAW DATA FOR EXPERIMENT 1.

\begin{tabular}{l|r|r|r}
\hline \multicolumn{1}{c|}{ Condition } & \multicolumn{1}{|c|}{ Belt1-D } & \multicolumn{1}{c|}{ Belt2-C } & \multicolumn{1}{c}{ Visual only } \\
\hline \hline Duration $(\mathrm{s})$ & $29,06 \pm 3,36$ & $21,39 \pm 4,79$ & $22,89 \pm 13,71$ \\
\hline Mean velocity $(\mathrm{mm} / \mathrm{s})$ & $23,07 \pm 4,37$ & $29,67 \pm 2,79$ & $37,19 \pm 1,82$ \\
\hline Mean error $(\mathrm{mm})$ & $4,80 \pm 0,44$ & $3,01 \pm 0,16$ & $2,90 \pm 0,21$ \\
\hline Maximum distance $(\mathrm{mm})$ & $16,51 \pm 1,69$ & $10,83 \pm 0,61$ & $10,65 \pm 1,23$ \\
\hline \hline
\end{tabular}

\section{B. Experiment 2}

Similar ANOVAs in repeated measurements (2 conditions $\mathrm{x} 6$ trials) were computed for each criterion (raw data are given in table II). The order effect was not significant (as expected) (all $p>.25$ ). All computed criterions, except duration $(p>.25)$, revealed significant differences between tactile conditions (Belt1-D and Belt2-C groups with respectively 2 and 7 subjects) and Visual Only condition (mean velocity: $F(1,8)=7.10 ; p<.05$; mean error: $F(1,8)=$ $6.72 ; p<.05$; maximum distance: $F(1,8)=7.87 ; p<.05$ and time looking navigation screen: $F(1,3)=11.64 ; p<$ $.05)$.

TABLE II

SUMMARY OF RAW DATA FOR EXPERIMENT 2.

\begin{tabular}{l|c|c|c}
\hline \multicolumn{1}{c|}{ Condition } & Tactile & Visual only & sig. \\
\hline Duration (s) & $88,85 \pm 21,89$ & $88,63 \pm 33,25$ & \\
\hline Mean velocity $(\mathrm{mm} / \mathrm{s})$ & $10,65 \pm 1,56$ & $14,12 \pm 2,71$ & $*$ \\
\hline Mean error $(\mathrm{mm})$ & $7,96 \pm 0,90$ & $13,37 \pm 3,24$ & $*$ \\
\hline Maximum distance $(\mathrm{mm})$ & $32,06 \pm 4,46$ & $52,72 \pm 17,67$ & $*$ \\
\hline Time looking navigation $(\%)$ & $49,75 \pm 12,05$ & $88,01 \pm 4,83$ & $*$ \\
\hline \hline
\end{tabular}

\section{DISCUSSION, CONCLUSIONS AND FUTURE WORKS}

The present study examined whether vibrotactile guidance can bring beneficial clues to realize a complex task of trajectory following, as encountered in skull base surgery. The results from experiment 1 showed a strong difference between our two guidance paradigms. The Belt1 paradigm revealed to be significantly different from Belt 2 and visual control conditions, with performances that tend to be lowered, suggesting a mismatch to the underlying mental representation. Belt2 paradigm showed equivalent performances as control condition. The lack of participants choosing Belt1$\mathrm{C}$ or Belt2-D suggests a cognitive inadequacy between coding and guidance paradigm. The results of the second experiment on a simulated skull base surgery showed a significant reduction of spatial errors (mean and maximum errors), a decrease of mean velocity, a significant reduction of the need for visual feedbacks (percentage of time looking at the navigation screen) and no significant differences in the duration, for the tactile guidance condition as compared to the control condition. It seems therefore that the proposed tactile guidance, even after a short familiarization period, can efficiently provide the localization information that are usually brought by the navigation systems, as emphasized by the reduction of the time passed to look at visual localization information. The interest for skull base surgery, and more generally speaking for complex on-line trajectories following, can be important with the objective to reduce the distance errors and to let the surgeon focus his/her attention to the operative field. However, this research only focused on a comparison between tactile guidance and the usual way of performing skull base surgery. A confrontation to other visual guidance [7] might precise the efficiency of such tactile guidance. We also need to investigate the target population (i.e. surgeons) to see if these promising results are confirmed. For information, preliminary results with one surgeon tend to confirm the positive effect of our tactile guidance. Other in situ experiments should also be conducted but the implementation implies further developments as concerns the tool tracking.

In conclusion, a surgery simulator was used to assess if a vibrotactile guidance could be implemented for complex trajectories following task. Two guidances paradigms were proposed based on two vibrotactile belts, showing significant differences in performances for naïve participants. Results seem to be very encouraging for an application in skull base surgery but further experiments need to be conducted on experts to be more specific about the effects of this tactile guidance in real operating conditions.

\section{REFERENCES}

[1] J. Bluteau. Évaluation des effets de l'ajout d'interfaces haptiques sur le suivi manuel de trajectoires. Phd thesis, Grenoble Université, France, June 2010.

[2] M. Brell, D. Rokamp, and A. Hein. Fusion of vibrotactile signals used in a tactile display in computer aided surgery. In Haptics: Perception, Devices and Scenarios, volume 5024 of Lecture Notes in Computer Science, pages 383-388. Springer Berlin / Heidelberg, 2008.

[3] M. Eriksson, H. Flemmer, and J. Wikander. A haptic and virtual reality skull bone surgery simulator. Eriksson, M., Flemmer, H., and Wikander, J. (2005). "A Haptic and Virtual Reality Skull Bone Surgery Simulator," Proceedings of World Haptics, March, Pisa, Italy., 2005.

[4] A. P. George and R. De. Review of temporal bone dissection teaching: how it was, is and will be. The Journal of Laryngology \& Otology, $1: 1-7,2009$.

[5] P. Merloz, J. Tonetti, L. Pittet, M. Coulomb, S. Lavallee, J. Troccaz, P. Cinquin, and P. Sautot. Computer-assisted spine surgery. Computer Aided Surgery, 3(6):297-305, 1998.

[6] D. Morris, C. Sewell, F. Barbagli, K. Salisbury, N.H. Blevins, and S. Girod. Visuohaptic simulation of bone surgery for training and evaluation. IEEE Computer Graphics and Applications, 26(6):48-57, Nov.-Dec. 2006.

[7] P. Mozer, A. Leroy, Y. Payan, J. Troccaz, E. Chartier-Kastler, and F. Richard. Computer-assisted access to the kidney. International Journal of Medical Robotics and Computer Assisted Surgery, 1(4):5866, 2005.

[8] G Ng, P Barralon, S K W Schwarz, G Dumont, and J M Ansermino. Evaluation of a tactile display around the waist for physiological monitoring under different clinical workload conditions. Conf Proc IEEE Eng Med Biol Soc, 2008(NIL):1288-91, 2008.

[9] F. Robineau. Etude d'un dispositif de guidage de geste chirurgical de ponction par stimulation lectrotactile linguale. $\mathrm{PhD}$ thesis, Universit Joseph Fourier, France, 2009.

[10] F. Robineau, F. Boy, J.-P. Orliaguet, J. Demongeot, and Y. Payan. Guiding the surgical gesture using an electro-tactile stimulus array on the tongue: A feasibility study. IEEE Transactions on Biomedical Engineering, 54(issue 2), 2007.

[11] J.O. Vazquez-BuenosAires, Y. Payan, and J. Demongeot. Electrostimulation of the tongue as a passive surgical guiding system. International Conference on Advanced Robotics, ICAR'2003:pp. 638643., 2003. 